\title{
Large-Scale Brain Network Dynamics Supporting Adolescent Cognitive Control
}

\author{
Dominic B. Dwyer, ${ }^{1}$ Ben J. Harrison, ${ }^{1}$ Murat Yücel, ${ }^{1,2}$ Sarah Whittle, ${ }^{1,4}$ Andrew Zalesky, ${ }^{1,3}$ Christos Pantelis, ${ }^{1}$ \\ Nicholas B. Allen, ${ }^{4,5}$ and Alex Fornito ${ }^{1,2}$ \\ ${ }^{1}$ Melbourne Neuropsychiatry Centre, Department of Psychiatry, The University of Melbourne and Melbourne Health, Carlton South, Victoria, Australia \\ 3053, ${ }^{2}$ Monash Clinical and Imaging Neuroscience, School of Psychological Sciences and Monash Biomedical Imaging, Monash University, Clayton, \\ Victoria, Australia 3168, and ${ }^{3}$ Melbourne School of Engineering, The University of Melbourne, Victoria, Australia 3010, ${ }^{4}$ Melbourne School of Psychological \\ Sciences, The University of Melbourne, Victoria, Australia 3010, and ${ }^{5}$ Orygen Youth Health Research Centre, Centre for Youth Mental Health, The University of \\ Melbourne, Victoria, Australia 3010
}

Adolescence is a time when the ability to engage cognitive control is linked to crucial life outcomes. Despite a historical focus on prefrontal cortex functioning, recent evidence suggests that differences between individuals may relate to interactions between distributed brain regions that collectively form a cognitive control network (CCN). Other research points to a spatially distinct and functionally antagonistic system - the default-mode network (DMN) - which typically deactivates during performance of control tasks. This literature implies that individual differences in cognitive control are determined either by activation or functional connectivity of CCN regions, deactivation or functional connectivity of DMN regions, or some combination of both. We tested between these possibilities using a multilevel fMRI characterization of CCN and DMN dynamics, measured during performance of a cognitive control task and during a task-free resting state, in 73 human adolescents. Better cognitive control performance was associated with (1) reduced activation of CCN regions, but not deactivation of the DMN; (2) variations in task-related, but not resting-state, functional connectivity within a distributed network involving both the CCN and DMN; (3) functional segregation of core elements of these two systems; and (4) task-dependent functional integration of a set of peripheral nodes into either one network or the other in response to prevailing stimulus conditions. These results indicate that individual differences in adolescent cognitive control are not solely attributable to the functioning of any single region or network, but are instead dependent on a dynamic and context-dependent interplay between the CCN and DMN.

Key words: adolescence; cognitive control; fMRI; functional connectivity; graph theory; modularity

\section{Introduction}

Effectively controlling thought and behavior during adolescence challenges some individuals more than others. For instance, lower cognitive control ability at this time is associated with risktaking behavior, substance abuse, and mental disorders (Paus et al., 2008), and is linked to morbidity, mortality, and crime (Coleman, 2011). Conversely, higher cognitive control is correlated with positive outcomes, such as academic and vocational success

Received April 18, 2014; revised Aug. 19, 2014; accepted Sept. 6, 2014.

Author contributions: D.B.D., B.J.H., M.Y., N.B.A., and A.F. designed research; D.B.D., S.W., and N.B.A. performed research; D.B.D., B.J.H., A.Z., and A.F. analyzed data; D.B.D., B.J.H., M.Y., S.W., A.Z., C.P., N.B.A., and A.F. wrote the paper.

This research was supported by grants from the Colonial Foundation, the National Health and Medical Research Council (NHMRC; Australia, Program Grant 350241), and the Australian Research Council (Discovery grants DP0878136 and DP1092637). A.F. was supported by an NHMRC grant (1050504) and an Australian Research Council Future Fellowship (FT130100589). B.J.H. was supported by an NHMRC Clinical Career Development Fellowship (628509). S.W. and A.Z. were supported by NHMRC Career Development Fellowships (1007716 to S.W.; 1047648 to A.Z.). M.Y. was supported by an NHMRC Senior Research Fellowship (1021973). C.P. was supported by an NHMRC Senior Principal Research Fellowship (628386). We also thank the Royal Children's Hospital for assistance with acquiring the imaging data and the families for participating in the study.

The authors declare no competing financial interests.

Correspondence should be addressed to Dominic B. Dwyer, Melbourne Neuropsychiatry Centre, Lv. 3, Alan Gilbert Building, 161 Barry Street, Carlton, VIC, Australia 3053. E-mail: dwyerd@unimelb.edu.au.

DOI:10.1523/JNEUROSCI.1634-14.2014

Copyright $\odot 2014$ the authors $\quad 0270-6474 / 14 / 3414096-13 \$ 15.00 / 0$
(Caspi et al., 1998; Moffitt et al., 2011). Understanding the neural basis of individual differences in adolescent cognitive control is thus a critical goal of developmental neuroscience (Casey et al., 2008; Luna, 2009), but it has received very little research attention to date (Luna et al., 2010).

Despite a strong focus on the PFC during adolescence (Casey et al., 2008), recent research, mainly conducted in adults, links the capacity for cognitive control to two large-scale brain networks. The first is a network of frontal, parietal, cingulate, and subcortical regions showing increased activation during cognitive control tasks-referred to here as the cognitive control network (CCN; Cole and Schneider, 2007; Nee et al., 2007; Dosenbach et al., 2008). The second is the so-called default-mode network (DMN; Shulman et al., 1997), which includes posterior cingulate, medial frontal, and lateral parietal regions, and whose task-related deactivation is linked to faster and more accurate performance during the same experimental paradigms (Weissman et al., 2006; Persson et al., 2007; Eichele et al., 2008; Congdon et al., 2010). Further research in task-free, so-called resting-state conditions has shown coherent spontaneous activity within each of these networks (Greicius et al., 2003; Vincent et al., 2008), and also a strong anticorrelation between the networks (Fox et al., 2009). The behavioral importance of this antagonistic relation- 
ship is indicated by research showing that more consistent performance is associated with higher anticorrelation during task performance and at rest (Kelly et al., 2008).

Although the extent to which these findings apply to adolescents is unclear, they suggest that individual differences in adolescent cognitive control may be mediated by the following: (1) activation and/or functional connectivity of CCN regions, (2) deactivation and/or functional connectivity of DMN regions, or (3) a dynamic interplay between both systems. To test between these hypotheses, we used fMRI to perform a comprehensive, multilevel analysis to investigate how individual differences in cognitive control at 16 years of age relate to task-related activation, deactivation, and functional connectivity of the CCN and DMN measured during a validated cognitive control task (Bush and Shin, 2006). In addition, we examined whether similar relationships were apparent in the brain's spontaneous dynamics to determine whether cognitive control performance is best explained by the context-dependent engagement of task-relevant neural networks, or an invariant or intrinsic component of brain functional connectivity that persists across task and rest states (Fornito and Bullmore, 2010). Our findings indicate that adolescent control is not solely attributable to $\mathrm{CCN}$ or DMN function, but rather relies on a complex and task-dependent interplay between regions of both systems.

\section{Materials and Methods \\ Participants}

The data presented here were collected as part of the Adolescent Development Study (ADS), which was a longitudinal research project that has been described in detail previously (Whittle et al., 2008). In brief, the ADS investigated biological, psychological, and social risk factors for psychopathology from 12 to 19 years in a sample of 245 participants who were selected from community schools in the Victorian metropolitan region of Australia on the basis of two temperamental traits. Specifically, 2453 participants were originally screened using the Early Adolescent Temperament Questionnaire Revised (Ellis and Rothbart, 2001), and a subsample of 415 was selected to maximize the range of scores on the effortful control (EC) and negative affectivity (NA) temperament dimensions. Of these, 157 initially agreed to be involved in the longitudinal neuroimaging component of the study that occurred when the participants were 12,16, and 19 years old.

In the current study, we used data collected within a tight age range centered on 16 years because it is a quintessential adolescent period when most individuals have undergone puberty (Costello et al., 2011), but remain homogeneous with respect to school and living circumstances (Arnett, 2000). As a result of random subject attrition, neuroimaging data at this time point were available for 140 participants. Based on this reduced sample, in light of the selective sampling procedure used to select the original cohort of 415 individuals, we then ensured that the distributions of NA and EC in this subsample were normal using Shapiro-Wilk tests (EC: $\mathrm{W}=0.98, p=0.36$; NA: $\mathrm{W}=0.98, p=0.51$ ), and that there were no differences in the mean values of each temperament dimension when compared with the original screening cohort of 2453 participants (EC mean difference $=-0.1 ; t_{(1,149.79)}=-1.59, p=0.11$; NA mean difference $\left.=0.07, t_{(1,147.29)}=0.89, p=0.38\right)$. Combined, these factors alleviated any potential sampling biases associated with the original selective recruitment procedure.

Participants were excluded if diagnosed with a medical condition $(n=$ $5)$ or if taking psychotropic medication at the time of scanning $(n=4)$, if incidental radiological abnormalities had been detected $(n=4)$, if clinical assessment with the Kiddie-Schedule for Affective Disorders and Schizophrenia revealed a current or past diagnosis of DSM-IV mental illness $(n=10)$, or if they had consumed $>4$ standard alcoholic drinks the night before scanning or illicit psychoactive substances in the preceding week $(n=3)$. Regarding fMRI assessments, participants were also excluded if there were acquisition problems (i.e., scanner malfunction,

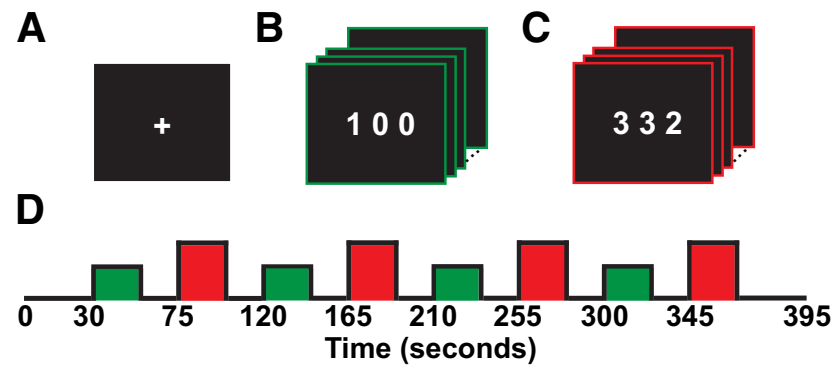

Figure 1. The MSIT is conducted using a block design with three main conditions: resting fixation, congruent stimuli, and incongruent stimuli. $\boldsymbol{A}$, Resting fixation is the presentation of a cross-hair. $\boldsymbol{B}$, The congruent condition involves responding to a target number that is congruent to the finger position on a button box. $C$, During the incongruent condition, the finger response differs from the location of the target on screen and the target is flanked by two other numbers. $D$, The experimental time course is depicted as an alternating block design with interleaved $15 \mathrm{~s}$ resting fixation periods between each 30 s block of congruent (green, $\boldsymbol{B}$ ) and incongruent (red, C) trials.

interference from ferrous metals, task timing or other errors, or poor visual acuity; $n=20$ ), if head motion exceeded $3 \mathrm{~mm}$ translation or 3 degrees rotation during the task or in the resting state $(n=13)$, if their accuracy during incongruent trials of the behavioral task was $<80 \%$ ( $n=$ $4)$, if they were left-handed $(n=2)$, or if they reported that they slept during the resting-state scan $(n=5)$. Accounting for three subjects who fulfilled overlapping exclusion criteria, a total of 67 subjects were excluded.

The final sample was of normal intelligence as measured by the Wechsler Abbreviated Scale of Intelligence (Wechsler, 1999), gender balanced, and within a tight age range centered on 16 years [age (mean years; $\mathrm{SD})=16.46(0.54)$; gender $($ male:female $)=36: 37 ; \mathrm{IQ}=105.2(14.45)]$. This tight age range reduced the effects of maturation on cognitive control and brain network organization.

\section{Experimental paradigm}

Each participant performed a version of the Multi-Source Interference Task (MSIT), which was administered using Presentation software (version 0.70; http://www.neurobs.com) and viewed via a head-coil mirror on a screen at the subject's feet. The MSIT is an established cognitive control fMRI paradigm designed to activate the $\mathrm{CCN}$ at a single-subject level by combining elements of the classical Simon and Eriksen flanker paradigms (Bush and Shin, 2006; Fig. 1) and has been extensively used in previous research (Matthews et al., 2007; Seminowicz and Davis, 2007; Harrison et al., 2007a,b; Yücel et al., 2007a,b,c; Bush et al., 2008; Karlsson et al., 2009; Stern et al., 2009; Brown et al., 2010; Rieckmann et al., 2011). In brief, subjects place their index, middle, and ring fingers on buttons that correspond to the numbers 1,2 , and 3 , respectively. They are then shown a row of three numerals, two of which are matching (e.g., "1 00 "), and they are required to press the button that corresponds to the unmatched number. During congruent trials, the unmatched number is positioned in the same spatial location as the finger response (e.g., "1 00 "), but in interference trials it is moved to other locations (and flanked by distracting numbers) to induce finger-to-number incongruence (e.g., "2 12 "). The task is organized in a block design with eight alternating blocks of $30 \mathrm{~s}$ congruent and incongruent trials. For this experiment, the duration of each trial was $2000 \mathrm{~ms}$ with stimulus presentation lasting either until this time or a when response was recorded. The intertrial interval was $500 \mathrm{~ms}$. Blocks of $30 \mathrm{~s}$ visual fixation were interleaved between congruent and incongruent blocks (following Harrison et al., 2007b). Task performance was measured using a conventional interference reaction time (RT) score, calculated by computing the difference between incongruent and congruent trials, where lower scores indicate less interference and thus better control performance. MSIT response accuracy was also calculated for each participant by summing the commission errors during congruent and incongruent conditions, and then representing these as a percentage of trials overall. 


\section{Neuroimaging acquisition and preprocessing}

MRI was performed for each participant in a single session with a $3 \mathrm{~T}$ Siemens Magnetom Trio B15 whole-body scanner at the Royal Children's Hospital, Victoria, Australia. Subjects' heads were fixed using foam padding and a 32-channel head coil. Resting-state and task fMRI were performed using single-shot gradient-recalled EPI providing $\mathrm{T} 2{ }^{\star}$ weighted BOLD contrast. To minimize prior task effects (Barnes et al., 2009; Gordon et al., 2014), resting-state fMRI was conducted before task performance with the following parameters: TR $1400 \mathrm{~ms}$, TE $30 \mathrm{~ms}$, flip angle $90^{\circ}$, FOV $210 \mathrm{~mm}$, voxel size $3.3 \times 3.3 \times 5.0 \mathrm{~mm}$, 24 slices, 510 whole-brain volumes. MSIT parameters were as follows: TR $2400 \mathrm{~ms}$, TE, $40 \mathrm{~ms}$, flip angle $90^{\circ}$, FOV $210 \mathrm{~mm}$, voxel size $3.3 \times 3.3 \times 3.0 \mathrm{~mm}, 36$ slices, 157 whole-brain volumes. Structural images were acquired as a gradient echo volumetric acquisition sequence (TR $1900 \mathrm{~ms}$, TE $2.24 \mathrm{~ms}$, FOV $230 \mathrm{~mm}$ ) to obtain $176 \mathrm{~T} 1$-weighted contiguous $0.9 \mathrm{~mm}$ slices $(0.9 \times 0.9 \times 0.9 \mathrm{~mm})$.

Image preprocessing and analysis were performed using Statistical Parametric Mapping toolbox (SPM8; www.fil.ion.ucl.ac.uk/spm/software/ spm8/) on a Linux computing cluster running MATLAB R2007a (MathWorks). Preprocessing involved magnetic field inhomogeneity correction and realignment, followed by spatial normalization using the Diffeomorphic Anatomical Registration using Exponentiated Lie Algebra (DARTEL; Ashburner, 2007) toolbox. Each subject's EPI volumes were unwarped using their field maps and were realigned using least-squares minimization with a six-parameter (rigid body) spatial transformation with the middle image as a reference. Spatial normalization to the MNI template was achieved via the DARTEL procedure, which is a nonlinear method with three main steps, as described previously (Ashburner, 2007): (1) within-subject registration of the EPI to T1, (2) deformation of the T1 to a sample-specific template, and (3) the use of the deformations to normalize the EPI to standard space. For this study, a template was constructed using all T1 images from the original neuroimaging sample $(n=140)$ after visual inspection. The images were then smoothed with an $8 \mathrm{~mm}$ full-width at half-maximum Gaussian kernel. At each processing stage, all images were quality controlled via visual inspection.

\section{Network mapping and region of interest identification}

Given that the CCN and DMN can be defined using task-related or resting-state maps (Shulman et al., 1997; Greicius et al., 2003), we used both to define ROIs designed to comprehensively sample key elements of each network. Specifically, DMN regions were defined as those regions either deactivated by the MSIT or showing significant positive functional connectivity with a seed region in the posterior cingulate cortex (PCC); CCN regions were defined as those regions either activated by the MSIT or showing significant negative functional connectivity (anticorrelation) with the PCC seed. All identified ROIs from both task and resting-state maps were then used in further analyses of activation, connectivity, and modularity. We adopted these methods as a simple alternative to multivariate techniques (Calhoun et al., 2001; Smith et al., 2009) to maintain consistency with prior work (Greicius et al., 2003; Fox et al., 2005; Kelly et al., 2008) and ensure that our CCN and DMN nodes sampled two networks that, by definition, showed some degree of functional antagonism during either rest or task.

Task-related mapping. GLM, as implemented in SPM8 (http://www.fil. ion.ucl.ac.uk/spm/software/spm8/), was used to define the task-related CCN (CCN-task) and the task-related DMN (DMN-task). The fixation, congruent, and incongruent task blocks were coded as individual regressors, convolved with a hemodynamic response function and incorporated as covariates in a GLM that was fitted on a voxelwise basis to the measured BOLD signal. The data were high-pass filtered $(1 / 128 \mathrm{~Hz})$ and temporal autocorrelations were corrected using a first-order autoregressive model. For each participant, the CCN was defined as regions showing statistically significant activation during incongruent trials compared with congruent trials (Bush and Shin, 2006). The DMN was defined as areas showing significantly greater activation during the fixation compared within congruent condition, as per previous research (Harrison et al., 2007b). Group-level statistical significance was determined using second-level random-effects one-sample $t$ tests. Model parameters were estimated using Restricted Maximum Likelihood (ReML). Statistical sig- nificance was defined in the group maps using a false discovery rate (FDR) threshold of $p=0.05$ (cluster extent of 20 voxels) and visualized on a population-average, landmark- and surface-based template using Caret (v5.62; http://brainvis.wustl.edu/wiki/index.php/Caret:About).

Resting-state mapping. In the resting-state condition, the $\mathrm{CCN}$ and DMN were defined using well established seed-based techniques (Fox et al., 2005, 2009; Kelly et al., 2008; Shehzad et al., 2009; Cauda et al., 2011). For each participant, the whole-brain resting-state functional volumes were detrended and bandpass filtered $(0.008<f<0.08 \mathrm{~Hz})$. Subjectspecific activity time courses were then extracted from an $8 \mathrm{~mm}$ spherical ROI placed in the midline area of the PCC using previously defined, approximated coordinates (MNI: [0 - 50 30]; Fox et al., 2009). Given considerable functional heterogeneity of the $\mathrm{PCC} /$ precuneus region (Margulies et al., 2009), the decision to use this specific seed was based on our aim to replicate the CCN and DMN networks as they have been previously reported (Fox et al., 2005, 2009). This seed time course was used as a regressor in a GLM, with additional nuisance covariates that included the mean signal from thresholded MNI masks of the white matter (WM; 90\%), CSF (70\%), and the whole-brain volume (WBV; $70 \%$ ) using SPM8 as well as six head-motion parameters (three translation, three rotation). Positive and negative correlations were modeled independently to map DMN and CCN regions for each subject, respectively. Group-level analysis maps were generated via a random effects, one-sample $t$ test and ReML estimation. Statistical significance was defined using a whole-brain corrected threshold of FDR $=0.05$ (cluster extent of 20 voxels) using Caret (v5.62).

ROI identification. To comprehensively sample the CCN and DMN, we generated spherical ROIs centered on activation/deactivation/connectivity maxima in the CCN and DMN maps derived from both the task and resting-state analyses. To adequately separate statistically significant clusters, resting-state maps were thresholded at an FWE rate of 0.001 and the MSIT maps were thresholded at FWE 0.01 (both with a cluster extent of 20 voxels) - this adaptive thresholding approach was necessary to account for $t$ score magnitude differences between the activation and connectivity maps. ROIs were then generated as $6 \mathrm{~mm}$ spheres centered on the voxel with the highest $t$ score in each remaining cluster for each map. To avoid overlap between ROIs, foci whose central points were within 8 $\mathrm{mm}$ of each other in Euclidean space were identified $(n=12)$, and a new focus was created at an equidistant location between them. In total, 73 ROIs were identified (Fig. 2) and were labeled according to their network of origin (Table 1).

\section{Testing correlations between MSIT performance and regional task-related activation, task-related functional connectivity, and resting-state functional connectivity}

Given the large degree of interindividual differences in the RT interference effect (see Results), we focused on this measure in further analysis by specifically examining its relationship to activation and functional connectivity of each of the 73 ROIs extracted from the CCN-task, DMN-task, CCN-rest, and DMN-rest.

Task-related activation analysis. For each of the 73 ROIs, mean parameter estimates encoding the magnitude of activation/deactivation in incongruent versus congruent trials of the MSIT were extracted and correlated with interference RT using Spearman's rank correlation and permutation testing (5000 permutations) to account for non-normality of the data. Only results that survived an FDR-corrected threshold of $p=$ 0.05 were declared significant (Benjamini and Hochberg, 2000).

Task-related functional connectivity analysis. To measure task-related pairwise functional connectivity, we used a previously described correlational psychophysiological interaction (cPPI) procedure (Fornito et al., 2012; http://www.nitrc.org/projects/cppi_toolbox). Briefly, each region's activity time course recorded during the MSIT was deconvolved (Gitelman et al., 2003), multiplied by a task regressor modeling activity increases in incongruent versus congruent trials, and then reconvolved to obtain a region-specific PPI term reflecting task-related modulations of neural activity in that region. Pairs of regional PPI terms were then correlated while partialing the effects of each region's original time course, the incongruent $>$ congruent task regressor, head-motion parameters, and the mean signal taken from WM, CSF, and WBV masks as defined 
A
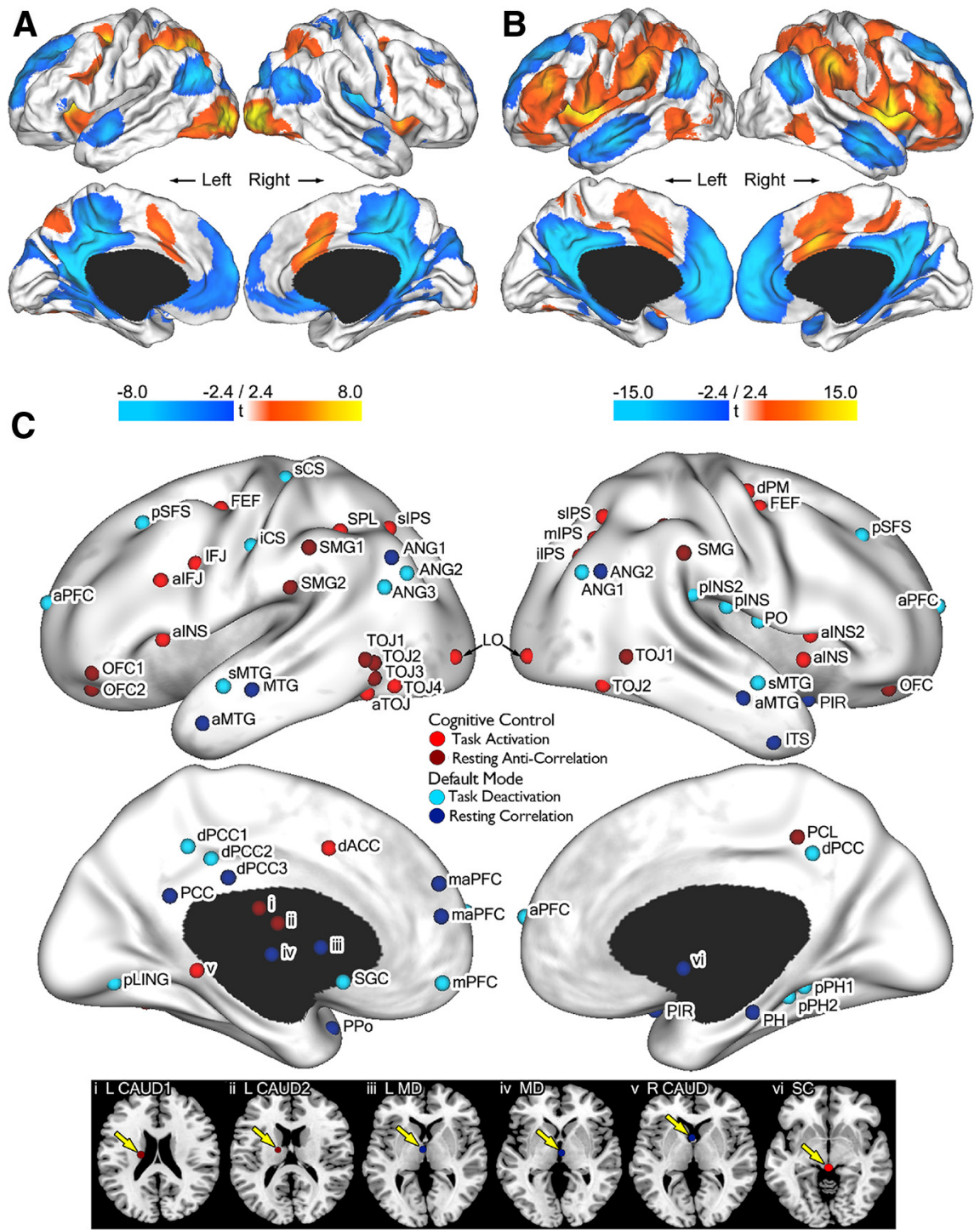

Figure 2. Identification of ROls from task activation and resting-state functional connectivity mapping of the CCN and DMN. $\boldsymbol{A}$, Task maps showing CCN activations during incongruent trials of the MSIT compared with congruent trials (yellow), and DMN deactivations when incongruent trials were compared with resting-fixation blocks (blue). $\boldsymbol{B}$, Resting-state maps showing positive (blue) and negative (yellow) functional connectivity with a posterior cingulate seed region (MNI: [0 - 50 30]), representative of the DMN and CCN, respectively. $\boldsymbol{C}$, Anatomical location of 73 seed regions drawn from the maps presented in $\boldsymbol{A}$ and $\boldsymbol{B}$. Subcortical seed regions (i-vi) depicted below. Node labels defined in Table 1.

above. Partialing out these effects isolates context-dependent functional connectivity between regions, as distinct from task-unrelated, spontaneous connectivity, and coactivation effects (Friston, 1994; Fornito et al., 2012). Before analysis, extreme ( $>4$ SD) outliers in all time courses were replaced via linear interpolation of adjacent time points.

Resting-state functional connectivity analysis. To measure resting-state pairwise functional connectivity, each region's time series was extracted and orthogonalized with respect to six head-motion parameters (rotation and translation) and the WM, CSF, and WBV masks. Functional connectivity was then estimated as the Spearman's correlation between each possible pair of regional activity time courses.

Testing the relationship between functional connectivity and performance. The resting-state and task-evoked functional connectivity analyses generated two $73 \times 73$ functional connectivity matrices (one for rest and one for task) per individual. We tested for associations between behavior and functional connectivity at the level of each of the $N(N-$ 1) $/ 2=2628$ pairwise connections in the network and used the networkbased statistic (NBS; Zalesky et al., 2010; http://www.nitrc.org/ projects/nbs) to identify subnetworks of functional connections showing covariation with individual differences in interference RT, while controlling type I error rate. There were four main steps: (1) a Spearman correlation was computed between the interference effect and functional connectivity at each of the 2628 pairwise connections; (2) the resulting brain-behavior association matrix was binarized by applying a primary threshold of $p<0.01$, uncorrected $(p<$ $0.01=1 ; 0$ otherwise); (3) a breadth-first search was used to compute the size of connected components (sets of nodes that can be linked by suprathreshold links) in the matrix; and (4) the data were permuted 10,000 times to generate an empirical null distribution of maximal component sizes, which was then used to determine a corrected $p$ value for the observed component sizes relative to this empirical null. Only components surviving a corrected component-wise threshold of $p<0.05$ were declared significant.

\section{Modularity analysis}

To better understand changes in functional network architecture between rest and task conditions, we used established graph theoretic methods to effect a data-driven decomposition of each individual's resting-state and taskrelated networks into modules: subsets of regions showing higher connectivity with each other than with other areas (Newman, 2006; Rubinov and Sporns, 2011). A consensus clustering technique (Rubinov and Sporns, 2011; Fornito et al., 2012; Lancichinetti and Fortunato, 2012) was then used to define group-level representations of network modular architecture separately for rest and task, and for high and low performers on the MSIT, as determined by a median split of the sample based on interference RT (high, 341.66 ms; low, 509.31 $\mathrm{ms} ; Z=7.34, p<0.001)$

Individual-level modular decompositions. To decompose each participant's taskrelated and resting-state functional connectivity networks into distinct modules, we optimized a variant of the widely used Newman-Girvan (Newman, 2006) quality function $Q$, termed $Q^{*}$, which was proposed by Rubinov and Sporns (2011) to account for signed (e.g., positively and negatively correlated) edge weights. In short, the modified function tries to maximize the difference between the degree of observed intramodular connectivity and that expected by chance, while biasing against solutions that assign negatively correlated regions to the same module. This function was optimized using the Louvain algorithm (Blondeletal.,2008) asimplementedin thebrain connectivity toolbox(https:// sites.google.com/site/bctnet/). To deal with the known degeneracy of this method (Good et al., 2010), we iterated the algorithm 1000 times and used a consensus-based approach (van den Heuvel et al., 2008; Fornito et al., 2012; Lancichinetti and Fortunato, 2012) to identify the final solution for each individual. Specifically, for each iteration, we generated a co-classification matrix in which each $[i, j]$ element contained 1 if two nodes were classified in the same module and 0 otherwise. Note that we use the term classification here in reference to the assignment of a node into a module, not with reference to classifying some outcome variable of interest. We summed these matrices across the 1000 iterations and then ran a second decomposition of this consistency matrix. In this manner, nodes frequently co-classified in the same module across multiple iterations of the algorithm will be assigned to the same module in the final solution. 
Table 1. Labels of each ROI

\begin{tabular}{|c|c|c|c|c|c|}
\hline \multirow[b]{2}{*}{ Map } & \multirow[b]{2}{*}{ Label } & \multirow[b]{2}{*}{ Brain region } & \multicolumn{3}{|c|}{ Talairach } \\
\hline & & & $x$ & $y$ & $z$ \\
\hline \multirow[t]{20}{*}{$\mathrm{CCNt}$} & $\mathrm{dACC}$ & Dorsal anterior cingulate cortex & -2 & 12 & 46 \\
\hline & SC & Superior colliculus & 0 & -30 & -6 \\
\hline & LalFJ & Anterior inferior frontal junction & -40 & 24 & 22 \\
\hline & LaINS & Anterior insula cortex & -30 & 21 & 4 \\
\hline & LaTOJ & Anterior temporo-occipital junction & -44 & -56 & -8 \\
\hline & LFEF & Frontal eye field & -24 & -4 & 52 \\
\hline & LIFJ & Inferior frontal junction & -40 & 2 & 32 \\
\hline & LLO & Lateral occipital cortex & -26 & -90 & -4 \\
\hline & LsIPS & Superior intraparietal sulcus & -22 & -64 & 48 \\
\hline & LSPL & Superior parietal lobule & -33 & -45 & 44 \\
\hline & L TOJ3 & Temporo-occipital junction & -40 & -68 & -10 \\
\hline & RalNS & Anterior insula cortex & 40 & 14 & -6 \\
\hline & RaINS2 & Anterior insula cortex & 35 & 18 & 5 \\
\hline & R TOJ2 & Temporo-occipital junction & 40 & -64 & -10 \\
\hline & R mIPS & Medial intraparietal sulcus & 26 & -62 & 40 \\
\hline & RLO & Lateral occipital cortex & 30 & -92 & -4 \\
\hline & RFEF & Frontal eye field & 26 & -2 & 50 \\
\hline & RPMd & Dorsal premotor cortex & 32 & -4 & 64 \\
\hline & RilPS & Inferior intraparietal sulcus & 28 & -66 & 32 \\
\hline & RsIPS & Superior intraparietal sulcus & 26 & -64 & 52 \\
\hline \multirow[t]{15}{*}{$\mathrm{CCNr}$} & LCAUD1 & Caudate nucleus & -18 & -16 & 22 \\
\hline & LCAUD2 & Caudate nucleus & -16 & -8 & 16 \\
\hline & L CERE & Cerebellum & -26 & -66 & -26 \\
\hline & L OFC1 & Orbitofrontal cortex & -46 & 48 & -16 \\
\hline & LOFC2 & Orbitofrontal cortex & -26 & 44 & -14 \\
\hline & LSMG1 & Supramarginal gyrus & -64 & -32 & 20 \\
\hline & LSMG2 & Supramarginal gyrus & -54 & -38 & 42 \\
\hline & L T0J1 & Temporo-occipital junction & -52 & -64 & -10 \\
\hline & L TOJ2 & Temporo-occipital junction & -58 & -66 & -4 \\
\hline & L TOJ4 & Temporo-occipital junction & -52 & -58 & -4 \\
\hline & ROFC & Orbitofrontal cortex & 26 & 44 & -14 \\
\hline & RPCL & Paracentral lobule & 18 & -44 & 48 \\
\hline & RSMG & Supramarginal gyrus & 60 & -34 & 40 \\
\hline & RSMG2 & Supramarginal gyrus & 44 & -42 & 44 \\
\hline & R T0J1 & Temporo-occipital junction & 56 & -54 & -4 \\
\hline \multirow[t]{22}{*}{ DMNt } & $\mathrm{mPFC}$ & Medial prefrontal cortex & -1 & 54 & -8 \\
\hline & SGC & Subgenual cingulate cortex & 0 & 22 & -8 \\
\hline & LANG2 & Angular gyrus & -42 & -76 & 32 \\
\hline & LANG3 & Angular gyrus & -46 & -66 & 25 \\
\hline & LaPFC & Anterior prefrontal cortex & -16 & 68 & 20 \\
\hline & $\mathrm{LdPCC1}$ & Dorsal posterior cingulate cortex & 0 & -42 & 48 \\
\hline & $\mathrm{LdPCC} 2$ & Dorsal posterior cingulate cortex & -4 & -36 & 44 \\
\hline & LiCS & Inferior central sulcus & -40 & -14 & 36 \\
\hline & LpLING & Posterior lingual gyrus & -16 & -72 & -8 \\
\hline & LpSFS & Posterior superior frontal sulcus & -22 & 28 & 50 \\
\hline & LsCS & Superior central sulcus & -18 & -30 & 64 \\
\hline & L sMTG & Superior middle temporal gyrus & -58 & -4 & -16 \\
\hline & R ANG1 & Angular gyrus & 46 & -74 & 32 \\
\hline & RaPFC & Anterior prefrontal cortex & -12 & 70 & 12 \\
\hline & $\mathrm{RdPCC}$ & Dorsal posterior cingulate cortex & 6 & -48 & 44 \\
\hline & RpINS & Posterior insula cortex & 36 & -16 & 20 \\
\hline & RpINS2 & Posterior insula & 44 & -32 & 24 \\
\hline & RPO & Parietal operculum & 54 & -4 & 8 \\
\hline & RpPH1 & Posterior parahippocampal gyrus & 30 & -42 & -8 \\
\hline & $\mathrm{RpPH} 2$ & Posterior parahippocampal gyrus & 27 & -35 & -14 \\
\hline & RpSFS & Posterior superior frontal sulcus & 24 & 34 & 42 \\
\hline & RsMTG & Superior middle temporal gyrus & 56 & -4 & -16 \\
\hline \multirow[t]{10}{*}{ DMNr } & MD & Mediodorsal thalamus & 0 & -12 & 0 \\
\hline & LaMTG & Anterior middle temporal gyrus & -52 & 4 & -30 \\
\hline & LANG1 & Angular gyrus & -44 & -70 & 38 \\
\hline & $\mathrm{LdPCC} 3$ & Dorsal posterior cingulate cortex & 0 & -30 & 34 \\
\hline & L maPFC1 & Medial anterior prefrontal cortex & -6 & 54 & 32 \\
\hline & L maPFC2 & Medial anterior prefrontal cortex & 0 & 56 & 20 \\
\hline & L MD & Mediodorsal thalamus & -2 & 8 & 4 \\
\hline & L MTG & Middle temporal gyrus & -58 & -14 & -16 \\
\hline & LPCC & Posterior cingulate cortex & 0 & -54 & 28 \\
\hline & $\mathrm{LPP} 0$ & Planum polare & -36 & $\begin{array}{c}14 \\
\text { (Tabel }\end{array}$ & $\begin{array}{r}-24 \\
\text { tinues) }\end{array}$ \\
\hline
\end{tabular}

Table 1. Continued

\begin{tabular}{lllrrr}
\hline & & & \multicolumn{3}{l}{ Talairach } \\
\cline { 3 - 5 } Map & Label & Brain region & $x$ & $y$ & \multirow{2}{*}{$z$} \\
\hline R ANG2 & Angular gyrus & 46 & -64 & 30 \\
R CAUD & Caudate nucleus & 4 & 4 & -4 \\
R ITS & Inferior temporal sulcus & 50 & 4 & -34 \\
R MTG & Middle temporal gyrus & 60 & -8 & -22 \\
R PIR & Piriform cortex & 30 & 14 & -20 \\
R PH & Parahippocampal gyrus & 22 & -20 & -18 \\
\hline
\end{tabular}

Far left column specifies the statistical map used to Lcreate the ROl; i.e., each ROI was identified using either the task-related activation (CCNt), task-related deactivation (DMNt), resting-state positive (DMNr), or resting-state negative (CCNr) functional connectivity map. L, left; $R$, right.

Group-level modular decomposition. The two final consensus decompositions for each individual (one for task and one for rest) were passed to a second level to derive a group-level representation of network modularity based on a similar logic to the consensus approach used at the single-subject level. A similar approach has been used in prior work (van den Heuvel et al., 2008; Fornito et al., 2012). Specifically, a coclassification matrix was generated for each individual's final consensus decomposition separately for task and rest. These matrices were then summed across individuals to generate a sample-level consistency matrix for both rest and task networks. A high weight in elements of this matrix indicated that two nodes were frequently classified in the same module across individuals. As such, modular decomposition of this group-level consistency matrix ensured that nodes frequently co-classified together were likely to be assigned to the same module in the final solution. This analysis was run separately within the high and low MSIT performing groups during rest and task conditions.

By aggregating results across single-participant decompositions, our consensus-based approach allowed us to derive a group-level representation while also characterizing interindividual variability in network organization using classification consistency and diversity metrics. Such analyses are not possible when decomposition is performed on a groupaveraged correlation matrix. Consensus-based approaches have also been shown to yield more stable individual module solutions, given the known degeneracy of most graph theoretic module detection algorithms (Good et al., 2010; Lancichinetti and Fortunato, 2012).

The group consistency matrices for rest and task conditions were also used to understand the role of each node within the network. In particular, the consistency and diversity with which each node was classified into distinct modules was calculated using a technique suited for weighted, signed matrices (Rubinov and Sporns, 2011). Regions with high classification consistency, $z$, are consistently classified as belonging to the same module across participants and relative to other regions in that module - they are thus core elements of that module and support functional specialization. Regions with high classification diversity, $h$, have relatively equal probability of being classified into different modules across participants because their connectivity is more dispersed across modules-these regions thus play a role in functionally integrating distinct neural systems.

Statistical significance of modular solutions. For the high- and lowperforming subgroups, we tested whether the final solutions obtained via the above procedures were significantly more modular than expected by chance (i.e., they showed nonrandom modularity) in three ways. First, at the participant level, we compared the median $Q^{*}$ statistic observed in the sample to the median $Q^{*}$ value obtained from an ensemble of 73 randomized functional connectivity networks, in which each random network was matched to an individual empirical network for degree, strength, and sign distributions (Rubinov and Sporns, 2011). The randomization was repeated $10^{3}$ times to generate a null distribution of median $Q^{*}$ values against which the magnitude of the observed sample median $Q^{*}$ statistic during rest and task conditions was compared. The sample median $Q^{*}$ for both subgroups was deemed to be significantly higher than expected by chance when evaluated with respect to the null model during task performance $\left(Q^{*}\right.$ high $=0.52, p<0.001 ; Q^{*}$ low $=$ $0.518, p<0.001)$ and during the resting state $\left(Q^{*}\right.$ high $=0.54, p<0.001$; $Q^{*}$ low $\left.=0.539, p<0.001\right)$. 

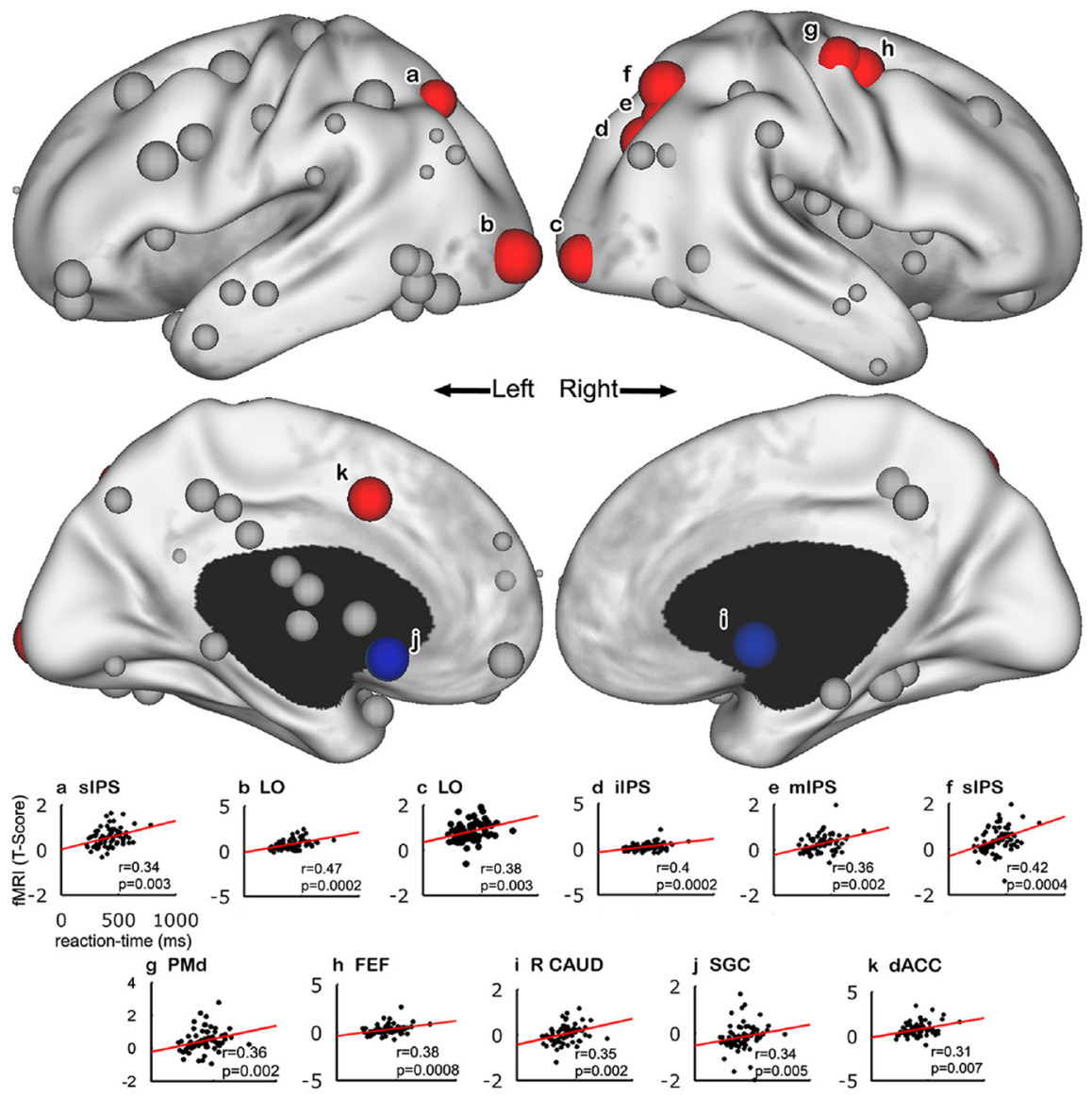

Figure 3. Activation correlates of interference RT. Colored nodes denote CCN (red) and DMN (blue) where regional activation was significantly correlated (FDR corrected) with interference RT. Gray nodes denote nonsignificant correlations. Node sizes are scaled according to correlation size (larger spheres $=$ higher correlation). Scatterplots $(\boldsymbol{a}-\boldsymbol{k})$ are displayed for all significant correlations. Node labels defined in Table 1. ilPs, inferior IPS; sIPS, superior IPS; mIPS, medial IPS; SGC, subgenual cingulate, R CAUD, right caudate.

The second method randomized at the level of each participant's consistency matrix, rather than functional connectivity matrix. The consistency matrix was randomized while again preserving degree and strength distribution (Rubinov and Sporns, 2011) $10^{3}$ times to generate a second ensemble of median $Q^{*}$ statistics under the null hypothesis, which was again used to evaluate the significance of the observed sample median $Q^{*}$. Once again, both the high- and low-performing subgroups showed statistically significant modularity using this method during task (high, $p<0.001$; low, $p=0.001$ ) and rest (high, $p<0.001$; low $p<0.001$ ) conditions.

Finally, to evaluate the significance of the group-level decomposition, we randomized each individual's final consensus co-classification matrix. We then summed these randomized matrices and ran the modular decomposition to obtain a group-level $Q^{*}$ under null expectations. We repeated this procedure $10^{3}$ times to generate a distribution of values against which the observed group-level $Q^{*}$ statistic was evaluated. Both high- and low-performing subjects showed statistically significant group-level modular organization during task $\left(Q^{*}\right.$ high $=0.29, p<$ $0.001 ; Q^{*}$ low $\left.=0.26, p<0.001\right)$ and rest $\left(Q^{*}\right.$ high $=0.28, p<0.001 ; Q^{*}$ low $=0.27, p<0.001)$ conditions.

Testing within-group and between-group differences in modular organization. We tested for differences in modular organization between rest and task, and high- and low-performing individuals, in three ways. First we used the Wilcoxon signed-rank test to compare $Q^{*}$ values across conditions or groups. $Q^{*}$ is a goodness-of-fit measure and can be used to index how modular a partition is relative to chance expectations. A difference between conditions or groups would suggest that one partition is significantly more modular than the other.

In a second analysis, we tested for differences in the partition distance between group-level modular solutions. This analysis is more sensitive to

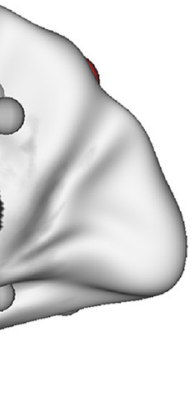

changes in the modular structure of a given partition, since two networks may show the same degree of overall modularity (i.e., $Q^{*}$ ) while having divergent patterns of node affiliations within different modules. Partition distance was quantified using the normalized variation of information (VIn), which quantifies the amount of information lost and gained in changing from one partition to another (VIn; Meilă, 2007). The statistical significance of changes in modular organization from rest to task was determined using a permutation procedure appropriate for repeated measures. Specifically, at each of $10^{3}$ iterations, the condition labels (i.e., rest or task) of the final coclassification matrices were swapped in a randomly chosen set comprising $\sim 50 \%$ of the individuals. These shuffled ensembles were then summed to generate a sample-level consistency matrix for each condition. These summed matrices were subjected to a modular decomposition and the VIn between these partitions was computed. The result was a null distribution of VIn values obtained under the null hypothesis of random labeling of task and rest datasets. This distribution was used to determine the significance of the observed VIn statistic comparing the rest and task group-level decompositions.

Finally, in a third analysis to determine whether the emergence of a third module in low-performing individuals was attributable to chance (Fig. 5), we tested for differences in the size of the third "intermediary" module between the high- and low-performing groups using a permutation approach for betweengroup comparisons. At each of $10^{3}$ iterations, we randomized each individual's final consensus co-classification matrix within the highand low-performing subgroups separately. We then summed the randomized matrices, performed a modular decomposition, and determined the number of nodes included in the third largest module. The difference in the size of the third module between the permuted data from each group was then calculated to generate a null distribution, against which the observed third module size difference of the high- and low-performing subgroups was compared using a one-tailed test of significance, based on the hypothesis that there should be a larger third module in low-performing subjects. This procedure was conducted separately for both rest and task conditions.

\section{Results}

\section{Control performance and task-related activation}

Mean interference RT was $422.04 \mathrm{~ms}\left(t_{(72)}=-33.54, p<0.001\right)$ with wide variability in performance noted across participants (SD = $107.6 \mathrm{~ms}$; range 220-782 ms). At FDR-corrected levels, task-related activation correlated with interference RT in 11 of 73 regions (Fig. 3). Two of these regions (the subgenual cingulate cortex and right caudate nucleus) were identified as part of the $\mathrm{DMN}$, whereas the remaining seven were localized to core components of the CCN that have been widely implicated in cognitive control performance (Cole and Schneider, 2007); namely, the dorsal anterior cingulate cortex (dACC), right frontal eye fields (FEF), right dorsal premotor cortex (PMd), and four regions spanning the bilateral intraparietal sulci (IPS). Significant correlations were also observed in bilateral lateral occipital (LO) cor- 

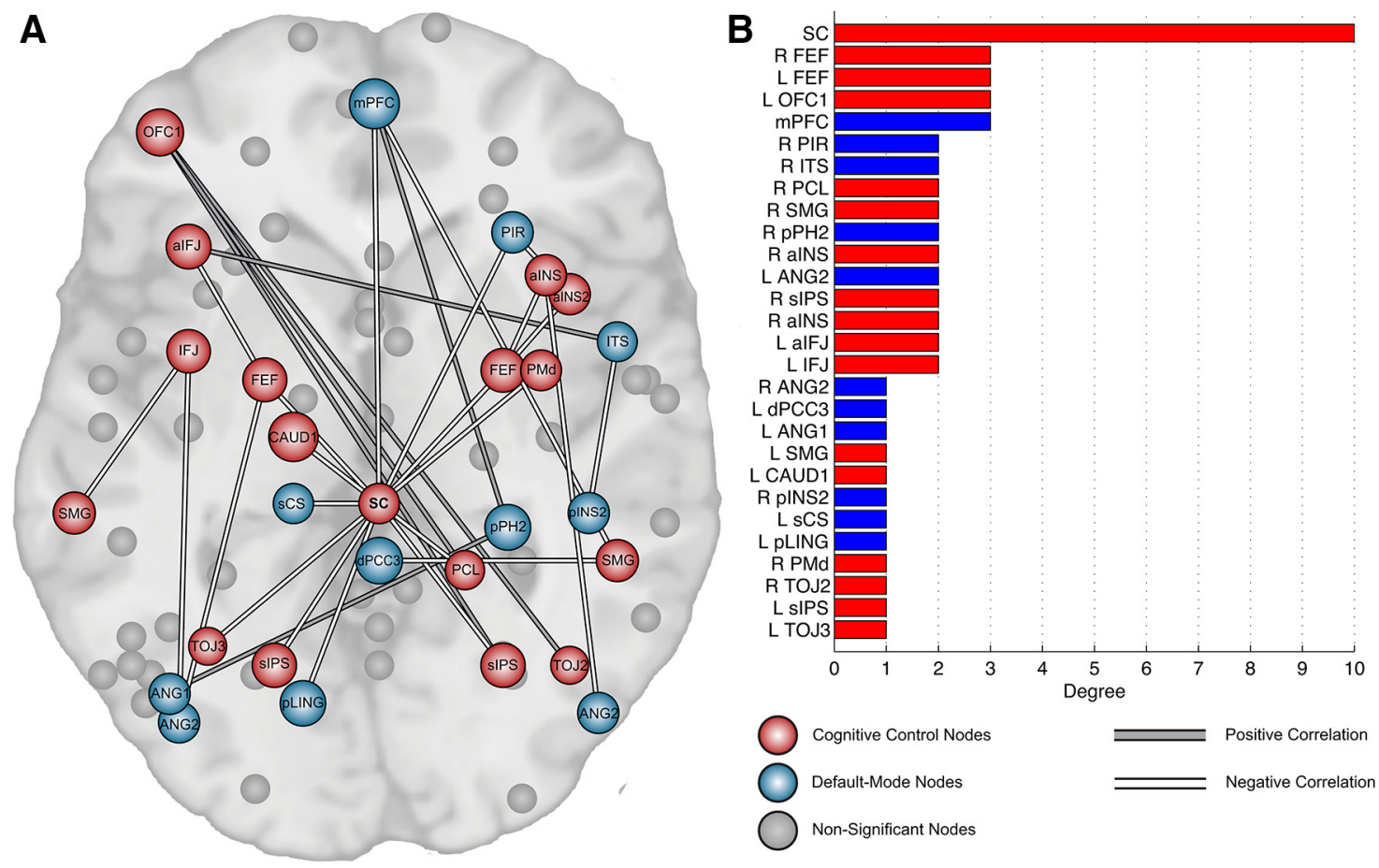

Figure 4. A, Interference RT is correlated with task-related functional connectivity in a distributed subnetwork of CCN (red) and DMN (blue) regions (legend, bottom right). Gray connections indicate that stronger functional connectivity was associated with higher interference RT (worse performance); white connections indicate that stronger functional connectivity was associated with lower RT (better performance).The z-dimension has been collapsed to aid visualization. $\boldsymbol{B}$, Number of connections (degree) possessed by each node in the performance-related network. Nodes are ordered from most connections (top) to least connections (bottom), and colored according to their network of origin (red, cognitive control; blue, default mode). Node locations displayed in Figure 2. Node labels defined in Table 1.

tices. Across all regions, reduced activation was associated with lower interference RT, thus reflecting better performance.

\section{Control performance and functional connectivity}

Consistent with a large body of activation studies (Luna et al., 2010), the above findings suggest that task-related activation of the CCN is the predominant neural correlate of individual differences in adolescent control performance. However, an unresolved question concerns the broader network context within which these regional activations occurred. For example, the results could reflect a tightly coupled subnetwork of CCN regions that support task performance, or the product of a more complex interplay between DMN and CCN regions. To test between these possibilities, we computed measures of functional connectivity between every ROI pair during the task-related and resting-state conditions and correlated these measures with interference RT. Subnetworks of functional connections showing statistically significant correlations ( $p<0.05$, corrected) with the MSIT interference effect during task-related and resting-state conditions were identified using the NBS (Zalesky et al., 2010).

Our analysis revealed a distributed subnetwork of taskrelated, but not resting-state, functional connectivity that correlated with interference RT ( $p=0.024$ and $p>0.85$, respectively; Fig. 4). Re-analysis of the resting-state data without the application of a low-pass filter to match the temporal filtering applied to the task data also failed to find a significantly correlated subnetwork $(p=0.18)$. The task-related network comprised 28 regions linked by 28 functional connections. Stronger functional connectivity was associated with better performance in 22 of these connections. More than $95 \%$ of these connections involved the CCN: $50 \%$ were intranetwork, linking different CCN elements with each other, while $45 \%$ were internetwork, linking
CCN and DMN regions. For the remaining 6/22 connections, stronger functional connectivity was correlated with poorer performance. Two of these connections (33\%) were intranetwork, linking distinct elements of the DMN; three were intranetwork CCN connections, and one linked CCN and DMN regions.

Investigation of the specific regions comprising the performancerelated subnetwork showed substantial involvement of areas implicated in visual attention: the superior colliculus (SC; 10 connections), left FEF (two connections), right FEF (three connections), and bilateral IPS (three connections; Fig. 4B). Each of these regions formed part of the $\mathrm{CCN}$, as defined here. Furthermore, four of the nine CCN regions showing a correlation between regional activation and interference RT (i.e., from the initial task-related activation analysis) were included in this subnetwork. We examined whether these two findings were related by computing the correlation between each region's functional connectivity strength (i.e., its summed connectivity with all other 72 regions, estimated separately for positive and negative connection weights) and its averaged parameter estimate for the activation contrast between congruent and incongruent MSIT trials. No significant correlations survived FDR correction, suggesting that the two measures made independent contributions to individual differences in task performance.

Given recent controversy (Saad, 2012), we repeated our analyses without correcting regional time courses for global signal fluctuations. The results were largely replicated. Specifically, task performance was correlated with a single significant component of task-related, but not resting-state, functional connectivity ( $p=0.0008$ and $p=0.81$, respectively). The task-related network largely overlapped with the network identified in the analysis of corrected data $(64 \%$ of $\mathrm{CCN}$ and $72 \%$ of DMN regions were 
A High performance rest

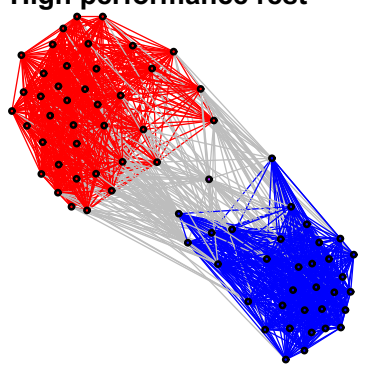

C Low performance rest

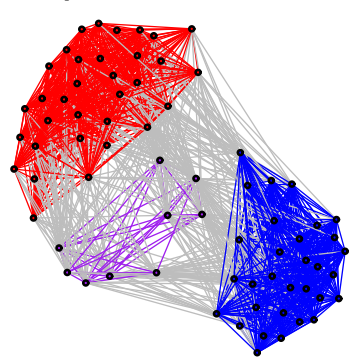

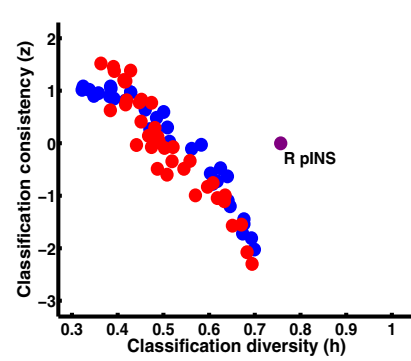

B High performance task

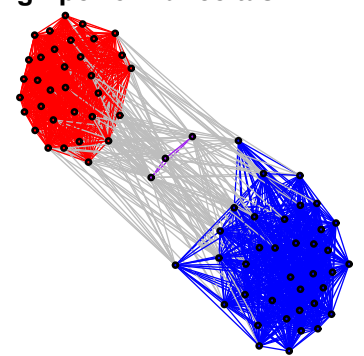

D Low performance task
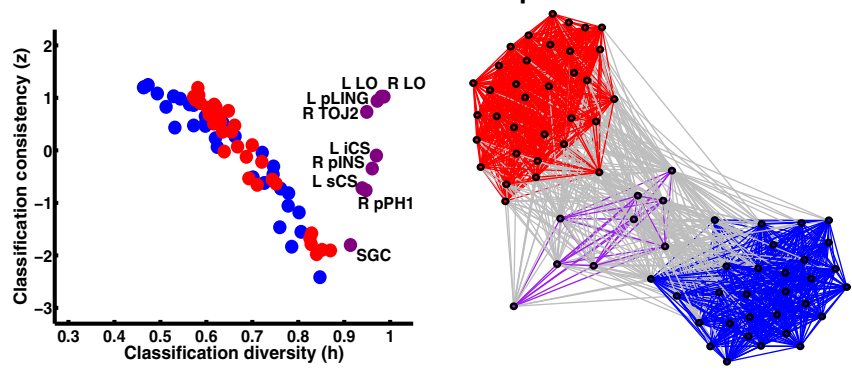
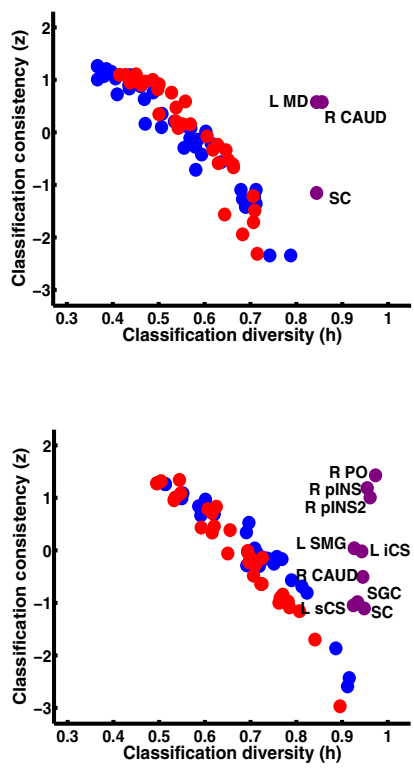

Figure 5. Better cognitive control ability is associated with bipartite brain functional organization. Fructherman-Reingold force-directed topological layouts of functional connectivity networks from high-performing participants during rest $(\boldsymbol{A})$ and task $(\boldsymbol{B})$ conditions, and from low-performing participants during rest $(\boldsymbol{C})$ and task $(\boldsymbol{D})$ conditions. Node color represents the modular affiliation of each region as defined by graph analytic modularity analysis (red, CCN-like module; blue, DMN-like module; purple, third, intermediary module). To assist visualization, graphs have been thresholded at $50 \%$ or greater co-classification frequency across participants. Intramodule connections are colored according to the parent module, intermodule connections are colored in gray. Scatterplots illustrate putative nodes roles in terms of classification consistency and diversity (see Materials and Methods). Brain organization in high performers was dominated by two large modules in both rest and task; low performers showed consistent evidence for three modules. Nodes in this third module showed on average higher classification diversity, pointing to variable module affiliation across subjects. Node locations displayed in Figure 2. R L0, right lateral occipital cortex; L L0 left lateral occipital cortex; L pLING, left posterior lingual gyrus; R T0J2, right temporo-occipital junction; L iCS, left inferior central sulcus; R pINS, right posterior insula cortex; L sCS, left superior central sulcus; R pPH1, right parahippocampal gyrus; SGC, subgenual cingulate cortex; R P0, right parietal operculum; R pINS, right posterior insula cortex; L SMG, left supramarginal gyrus; MD, mediodorsal thalamus; R CAUD, right caudate.

common between results). Prominent visual network involvement was also noted, with the SC, FEF, and IPS featuring prominently in both networks.

Collectively, these results indicated that better control performance was associated with: (1) greater task-dependent intranetwork functional integration of CCN regions; (2) greater task-dependent internetwork functional connectivity between the CCN and DMN; and (3) weaker task-dependent intranetwork integration of specific DMN regions, particularly medial prefrontal and lateral parietal cortices.

\section{Bipartite network organization supports better control performance}

The finding that interference RT correlates specifically with taskrelated, but not resting-state, functional connectivity suggests that adolescent control performance is subserved by a dynamic, state-dependent reconfiguration of CCN and DMN interactions, rather than a stable or intrinsic property of these systems. To further understand these state-dependent changes in functional network architecture, we investigated network modularity across rest and task conditions within subsamples of high- and lowperforming subjects (see Materials and Methods).

During both rest and task, two large modules dominated brain functional organization in high performers (Fig. 5). Both task and rest solutions were significantly more modular than comparable random networks using three different methods of randomization ( $p<0.001$; see Materials and Methods), and the degree of modularity, as indexed by the goodness-of-fit statistic $Q^{*}$ (Rubinov and Sporns, 2011), was consistent across both conditions $(Z=0.86, p=0.39)$. The first module showed considerable overlap with the $\mathrm{CCN}-$ i.e., $87 \%$ of nodes in this module identified during rest, and $100 \%$ during task belonged to the CCN (Table 1). The second module showed strong overlap with DMN nodes (97\% rest; $97 \%$ task). A third module, comprising only the posterior insula region, was identified during rest in high performers, indicating that this region had equal probability of being co-classified with either DMN or CCN nodes across individuals - a finding that is consistent with evidence that the functional coupling of the posterior insula with other systems is highly dependent on task context (Harrison et al., 2011). A small third module during task was also found, comprising the SC and two subcortical regions, thus further highlighting the role of visual attention in task performance. Furthermore, five regions were found to change their affiliation between CCN-like and DMNlike modules from rest to task conditions (Table 2). Combined, these task-related transitions were deemed to be significant when quantified with the variation of information statistic, which quantifies the partition distance between two different modular decompositions ( $V I n=0.15, p=0.001)$. Notably, all of the transitional nodes were derived from maps of task-related activation/ deactivation (Fig. 2A) rather than resting-state functional connectivity (Fig. $2 B$; see also Table 1 ), suggesting that they may variably couple with either the CCN or DMN depending on prevailing task demands. Thus, while overall modular organization was preserved across rest and task in high performers, small subsets of regions showed context-dependent coupling with one or the other system.

In contrast to high performers, low-performing individuals showed consistent evidence for a three-module solution during rest and task (Fig. 5). Both solutions were significantly more modular than expected by chance using three methods of randomization ( $p<0.001$; see Materials and Methods), and there 
Table 2. Modular affiliations of each ROI that changed from rest-to-task or was included in the third, intermediary module

\begin{tabular}{|c|c|c|c|c|c|c|}
\hline \multirow[b]{2}{*}{ Map } & \multirow[b]{2}{*}{ Label } & \multirow[b]{2}{*}{ Brain region } & \multicolumn{2}{|c|}{ High performers } & \multicolumn{2}{|c|}{ Low performers } \\
\hline & & & Rest & Task & Rest & Task \\
\hline \multirow[t]{4}{*}{$\mathrm{CCNt}$} & L LO & Lateral occipital cortex & $\ldots$ & $\ldots$ & Inter & $\ldots$ \\
\hline & R T0J2 & Temporo-occipital junction & $\ldots$ & $\ldots$ & Inter & $\cdots$ \\
\hline & RLO & Lateral occipital cortex & $\ldots$ & $\ldots$ & Inter & $\ldots$ \\
\hline & SC & Superior colliculus & DMNm & Inter & DMNm & Inter \\
\hline $\mathrm{CCNr}$ & LSMG1 & Supramarginal gyrus & $\ldots$ & $\ldots$ & $\ldots$ & Inter \\
\hline \multirow[t]{8}{*}{ DMNt } & L pLING & Posterior lingual gyrus & $\mathrm{CCNm}$ & $\cdots$ & Inter & $\ldots$ \\
\hline & $\mathrm{LsCS}$ & Superior central sulcus & $\mathrm{CCNm}$ & & Inter & Inter \\
\hline & LiCS & Inferior central sulcus & $\mathrm{CCNm}$ & . & Inter & Inter \\
\hline & R pPH1 & Posterior parahippocampal gyrus & $\ldots$ & $\cdots$ & Inter & $\ldots$ \\
\hline & RpINS & Posterior insula cortex & Inter & $\ldots$ & Inter & Inter \\
\hline & R PO & Parietal operculum & $\mathrm{CCNm}$ & & $\mathrm{CCNm}$ & Inter \\
\hline & R pINS2 & Posterior insula & $\mathrm{CCNm}$ & $\ldots$ & $\mathrm{CCNm}$ & Inter \\
\hline & SGC & Subgenual cingulate cortex & $\ldots$ & $\ldots$ & Inter & Inter \\
\hline \multirow[t]{2}{*}{ DMNr } & L MD & Mediodorsal thalamus & $\ldots$ & Inter & $\ldots$ & $\mathrm{CCNm}$ \\
\hline & RCAUD & Caudate nucleus & $\ldots$ & Inter & $\ldots$ & Inter \\
\hline
\end{tabular}

Map column specifies the statistical map used to create the ROl; i.e., each ROI was identified using either the task-related activation (CCNt), task-related deactivation (DMNt), resting-state positive (DMNr), or resting-state negative (CCNr) functional connectivity map. The last four columns identify the module affiliation of each node during rest and task in high and low performers separately. Modules were labeled as the $(\mathrm{CN}$-like module (CCNm), DMN-like module (DMNm), or a third, intermediary module ("inter"); ellipses represent agreement between the map used to create the ROI and the modular affiliation, e.g., $\mathrm{CCNt}$ and $\mathrm{CCNm}$.

was a significant reduction in the degree of network modularity during task $(Z=2.42$; $p=0.015)$. The nodes of one module overlapped substantially with the CCN (94\% rest; $97 \%$ task), the other with the DMN (97\% rest; 100\% task). The third "intermediary" module comprised a variable set of nodes across rest and task conditions (only 4/16 implicated regions were consistently assigned to this module across rest and task; Table 1). Accordingly, the partition distance between the rest and task solutions was significantly different ( $V I n=0.2, p<0.001$ ). Permutation tests indicated that the emergence of this third module in low performers was not attributable to chance during rest (high performers, one region; low performers, nine regions; $p<0.03$ ), or during task conditions at trend levels (high performers, three regions; low performers, nine regions; $p=0.07$ ). The transitional nodes belonging to the third module were largely derived from the analysis of task-related (de)activation (Fig. 2A), rather than resting-state functional connectivity (Fig. $2 B$ ). They were also the same regions showing variable module affiliation from rest to task in high performers (Table 2). Together, these findings suggest that these transitional regions are not part of the canonical CCN or DMN, but instead show variable coupling with either system based on prevailing task demands. To test this hypothesis, we computed an index of the diversity of each node's module affiliation across subjects (Fornito et al., 2012; see Materials and Methods). Nodes in this third module showed significantly higher classification diversity than nodes in the other two modules (task: Kruskal-Wallis $H(1)=23.354, p<0.001$; rest: $H(1)=$ 23.351, $p<0.001$; Figure $5 C, D$ ), indicating that they showed a variable pattern of module affiliation from individual to individual.

Collectively, these findings indicate that a bipartite division of the brain into modules resembling the CCN and DMN is associated with better control performance in adolescents, and that the dynamic engagement of noncore, transitional nodes into one or the other network plays a critical role in adapting this bipartite organization to changing task contexts. In particular, a failure to integrate these transitional regions into one of the two dominant systems is associated with poorer task performance.

\section{Discussion}

Individual differences in adolescent cognitive control are important for a diverse range of outcomes (Paus et al., 2008), though their neural bases have been elusive. Some studies have pointed to a prominent role for activation of PFC and other CCN regions (Pardo et al., 1990; Carter et al., 1998; Botvinick et al., 2001), others to deactivation of DMN areas (Weissman et al., 2006; Persson et al., 2007; Eichele et al., 2008), and yet others to functional interactions either within or between these systems (Kelly et al., 2008). The multilevel characterization presented here indicates that better control performance in adolescents is related to the following: (1) reduced task-evoked activation of CCN regions; (2) dynamic changes in task-related, but not resting-state, functional connectivity both within and between the CCN and DMN; and (3) the dynamic recruitment of noncore, transitional nodes into either the CCN or DMN in response to prevailing task demands.

Our findings confirm previous reports demonstrating that CCN activation supports cognitive control (Cole and Schneider, 2007; Nee et al., 2007; Roberts and Hall, 2008; Houdé et al., 2010), while also agreeing with developmental research demonstrating the counterintuitive result that reduced activation, in particular, is related to better performance (Luna et al., 2010). Commonly, an association between reduced activation and better performance is interpreted as reflecting enhanced neural efficiency (Luna et al., 2010). This interpretation may seem at odds with our finding that increased intranetwork functional connectivity of the $\mathrm{CCN}$ was also associated with better performance. However, studies of visual perception have found that greater interregional synchronization is associated with reduced regional activity, suggesting that enhanced synchronization increases processing efficiency and thus results in a reduced need for regional activation (Ghuman et al., 2008). As such, our finding of better performance being associated both with reduced activation and increased functional connectivity of CCN regions implies that efficient neural processing may involve distributing cognitive load across different brain regions, rather than placing too much burden on any single network element. A role for DMN regions in distributing this load is suggested as well, since our findings indicate that better control performance was also associated with increased internetwork connectivity between the CCN and DMN, in addition to reduced intranetwork integration of DMN elements. The lack of correlation between activation and connectivity measures observed in our study suggests that they make independent contributions to performance. Although the network determinants of regional variations in activation remain poorly understood, investigating how the maturation of longrange connections between large-scale networks such as the DMN and CCN (Fair et al., 2007, 2008; Dosenbach et al., 2010) relates to developmental changes in regional activation (and deactivation) will prove useful in better understanding their relative contributions to individual differences in cognitive performance.

The finding that better performance was associated with increased task-related functional connectivity between specific elements of the CCN and DMN is consistent with recent work demonstrating enhanced functional integration of these systems during certain task contexts (Leech et al., 2011; Fornito et al., 2012; Spreng and Schacter, 2012). In addition, high performers showed consistent evidence for a clear distinction between these two systems in terms of network modular structure, implying that strong functional segregation of the CCN and DMN was beneficial for task performance. These apparently contradictory 
findings can be reconciled by considering nodes that changed their module affiliation from rest to task. Specifically, a distinction can be made between a core set of regions comprising the canonical CCN and DMN (Shulman et al., 1997; Cole and Schneider, 2007), which were consistently classified into one or the other module across both rest and task in high and low performers (e.g., dACC for the CCN or the PCC for the DMN), and a set of transitional nodes that are not traditionally considered to be part of the CCN or DMN, and which changed their module affiliation across conditions and groups (e.g., SC and posterior insula cortex). These latter nodes were derived from maps of taskrelated activation or deactivation rather than resting-state functional connectivity, suggesting that their engagement with either the $\mathrm{CCN}$ or DMN is context dependent. In high performers, these regions rapidly integrated one or the other system, depending on prevailing stimulus conditions. In low performers, these regions failed to integrate with the CCN or DMN, forming a third, intermediary module.

Together, these findings suggest that better performance was associated with a delicate balance between functional segregation and integration; that is, a strong segregation of core elements of the canonical DMN and CCN, coupled with a dynamic integration of putative transitional or intermediary nodes with one or the other network in response to changing task demands. Similar results have been reported in recent analyses of nonstationary network dynamics recorded with resting-state magnetoencephalography, where the functional coupling between specific elements of the DMN and regions of other, cognitive control-related systems, was found to vary over time (de Pasquale et al., 2012). The findings also accord with past reports that better cognitive performance is associated with both stronger functional segregation of the CCN and DMN (Kelly et al., 2008), as well as stronger task-dependent integration between specific elements of these networks (Fornito et al., 2012), depending on the task at hand. An advantage of our approach is that it combines information from both resting-sate and task-related data to define the networks of interest. It is thus not biased by a single state (i.e., rest or task) or measure (connectivity or activation). However, alternative methods of network definition may facilitate a more fine-grained analysis of different subcomponents of the CCN (Fox et al., 2006; Vincent et al., 2008; Smith et al., 2009; Menon and Uddin, 2010; Power et al., 2011; Uddin et al., 2011; Fornito et al., 2012).

We did not identify any significant correlations between MSIT interference RT and resting-state functional connectivity. This finding indicates that adolescent control performance was specifically associated with context-dependent alterations of network dynamics, rather than an intrinsic property of spontaneous brain activity as putatively captured by resting-state fMRI (but see Fornito and Bullmore, 2010 and Harrison et al., 2011 for caveats). It also highlights the importance of distinguishing between resting-state and task-related functional connectivity measures for understanding individual differences in cognition (Fornito et al., 2012; for review, see Cocchi et al., 2013). One notable result was the heavy involvement of visual regions in both the activation and functional connectivity correlates of control performance. These visual areas, which included the SC, FEF, IPS, and LO, have been shown in nonhuman primates to support performance on antisaccade tasks that require inhibition of prepotent saccadic eye movements (Munoz and Everling, 2004). In our task, this visual response may have been related to the longer RTs during incongruent trials, since task stimuli terminated upon response and thus necessitated slightly longer periods of targeted visual fixation. However, we did not find significant ac- tivation of primary visual regions, such as V1, and there is limited evidence for the involvement of the antisaccade network during sustained attention processes (Schneider and Kastner, 2009). These findings thus point to an important role for top-down enhancement of visual attention mechanisms to specifically overcome task-related conflict during adolescence, consistent with theories emphasizing a role for prefrontal regions in top-down biasing of perceptual processes performed in posterior areas (Miller, 2000).

In summary, our multilevel analysis indicates that adolescent cognitive control is not merely a property of PFC function, nor is it solely attributable activity of CCN regions. Rather, it involves a delicate, context-dependent balance between functional segregation and integration of specific CCN and DMN elements. Our results draw attention to the complex neural basis of control abilities in teenagers and the large-scale reconfigurations of network architecture required to support optimal task performance.

\section{References}

Arnett JJ (2000) Emerging adulthood: a theory of development from the late teens through the twenties. Am Psychol 55:469-480. CrossRef Medline

Ashburner J (2007) A fast diffeomorphic image registration algorithm. Neuroimage 38:95-113. CrossRef Medline

Barnes A, Bullmore ET, Suckling J (2009) Endogenous human brain dynamics recover slowly following cognitive effort. PLoS One 4:e6626. CrossRef Medline

Benjamini Y, Hochberg Y (2000) On the adaptive control of the false discovery rate in multiple testing with independent statistics. J Educ Behav Stat 25:60-83. CrossRef

Blondel VD, Guillaume JL, Lambiotte R, Lefebvre E (2008) Fast unfolding of communities in large networks. J Stat Mech P10008. CrossRef.

Botvinick MM, Braver TS, Barch DM, Carter CS, Cohen JD (2001) Conflict monitoring and cognitive control. Psychol Rev 108:624-652. CrossRef Medline

Brown AB, Biederman J, Valera EM, Doyle AE, Bush G, Spencer T, Monuteaux MC, Mick E, Whitfield-Gabrieli S, Makris N, LaViolette PS, OscarBerman M, Faraone SV, Seidman LJ (2010) Effect of dopamine transporter gene (SLC6A3) variation on dorsal anterior cingulate function in attention-deficit/hyperactivity disorder. Am J Med Genet B Neuropsychiatr Genet 153B:365-375. CrossRef Medline

Bush G, Shin LM (2006) The Multi-Source Interference Task: an fMRI task that reliably activates the cingulo-frontal-parietal cognitive/attention network. Nat Protoc 1:308-313. CrossRef Medline

Bush G, Spencer TJ, Holmes J, Shin LM, Valera EM, Seidman LJ, Makris N, Surman C, Aleardi M, Mick E, Biederman J (2008) Functional magnetic resonance imaging of methylphenidate and placebo in attention-deficit/ hyperactivity disorder during the multi-source interference task. Arch Gen Psychiatry 65:102-114. CrossRef Medline

Calhoun VD, Adali T, Pearlson GD, Pekar JJ (2001) A method for making group inferences from functional MRI data using independent component analysis. Hum Brain Mapp 14:140-151. CrossRef Medline

Carter CS, Braver TS, Barch DM, Botvinick MM, Noll D, Cohen JD (1998) Anterior cingulate cortex, error detection, and the online monitoring of performance. Science 280:747-749. CrossRef Medline

Casey BJ, Getz S, Galvan A (2008) The adolescent brain. Dev Rev 28:62-77. CrossRef Medline

Caspi A, Wright BRE, Moffitt TE, Silva PA (1998) Early failure in the labor market: childhood and adolescent predictors of unemployment in the transition to adulthood. Am Sociol Rev 63:424-451. CrossRef

Cauda F, D’Agata F, Sacco K, Duca S, Geminiani G, Vercelli A (2011) Functional connectivity of the insula in the resting brain. Neuroimage 55:8-23. CrossRef Medline

Cocchi L, Zalesky A, Fornito A, Mattingley JB (2013) Dynamic cooperation and competition between brain systems during cognitive control. Trends Cogn Sci 17:493-501. CrossRef Medline

Cole MW, Schneider W (2007) The cognitive control network: integrated cortical regions with dissociable functions. Neuroimage 37:343-360. CrossRef Medline

Coleman JC (2011) The nature of adolescence. New York: Routledge.

Congdon E, Mumford JA, Cohen JR, Galvan A, Aron AR, Xue G, Miller E, 
Poldrack RA (2010) Engagement of large-scale networks is related to individual differences in inhibitory control. Neuroimage 53:653-663. CrossRef Medline

Costello EJ, Copeland W, Angold A (2011) Trends in psychopathology across the adolescent years: what changes when children become adolescents, and when adolescents become adults? J Child Psychol Psychiatry 52:1015-1025. CrossRef Medline

de Pasquale F, Della Penna S, Snyder AZ, Marzetti L, Pizzella V, Romani GL, Corbetta M (2012) A cortical core for dynamic integration of functional networks in the resting human brain. Neuron 74:753-764. CrossRef Medline

Dosenbach NU, Fair DA, Cohen AL, Schlaggar BL, Petersen SE (2008) A dual-networks architecture of top-down control. Trends Cogn Sci 12:99105. CrossRef Medline

Dosenbach NU, Nardos B, Cohen AL, Fair DA, Power JD, Church JA, Nelson SM, Wig GS, Vogel AC, Lessov-Schlaggar CN, Barnes KA, Dubis JW, Feczko E, Coalson RS, Pruett JR Jr, Barch DM, Petersen SE, Schlaggar BL (2010) Prediction of individual brain maturity using fMRI. Science 329: 1358-1361. CrossRef Medline

Eichele T, Debener S, Calhoun VD, Specht K, Engel AK, Hugdahl K, von Cramon DY, Ullsperger M (2008) Prediction of human errors by maladaptive changes in event-related brain networks. Proc Natl Acad Sci U S A 105:6173-6178. CrossRef Medline

Ellis L, Rothbart M (2001) Revision of the early adolescent temperament questionnaire. Biennial Meeting of the Society for Research in Child Development, Minneapolis, $\mathrm{MN}$.

Fair DA, Dosenbach NU, Church JA, Cohen AL, Brahmbhatt S, Miezin FM, Barch DM, Raichle ME, Petersen SE, Schlaggar BL (2007) Development of distinct control networks through segregation and integration. Proc Natl Acad Sci U S A 104:13507-13512. CrossRef Medline

Fair DA, Cohen AL, Dosenbach NU, Church JA, Miezin FM, Barch DM, Raichle ME, Petersen SE, Schlaggar BL (2008) The maturing architecture of the brain's default network. Proc Natl Acad Sci U S A 105:40284032. CrossRef Medline

Fornito A, Bullmore ET (2010) What can spontaneous fluctuations of the blood oxygenation-level-dependent signal tell us about psychiatric disorders? Curr Opin Psychiatry 23:239-249. CrossRef Medline

Fornito A, Harrison BJ, Zalesky A, Simons JS (2012) Competitive and cooperative dynamics of large-scale brain functional networks supporting recollection. Proc Natl Acad Sci U S A 109:12788-12793. CrossRef Medline

Fox MD, Snyder AZ, Vincent JL, Corbetta M, Van Essen DC, Raichle ME (2005) The human brain is intrinsically organized into dynamic, anticorrelated functional networks. Proc Natl Acad Sci U S A 102:9673-9678. CrossRef Medline

Fox MD, Corbetta M, Snyder AZ, Vincent JL, Raichle ME (2006) Spontaneous neuronal activity distinguishes human dorsal and ventral attention systems. Proc Natl Acad Sci U S A 103:10046-10051. CrossRef Medline

Fox MD, Zhang D, Snyder AZ, Raichle ME (2009) The Global signal and observed anticorrelated resting state brain networks. J Neurophysiol 101: 3270-3283. CrossRef Medline

Friston KJ (1994) Functional and effective connectivity in neuroimaging: a synthesis. Hum Brain Mapp 2:56-78. CrossRef

Ghuman AS, Bar M, Dobbins IG, Schnyer DM (2008) The effects of priming on frontal-temporal communication. Proc Natl Acad Sci U S A 105: 8405-8409. CrossRef Medline

Gitelman DR, Penny WD, Ashburner J, Friston KJ (2003) Modeling regional and psychophysiologic interactions in fMRI: the importance of hemodynamic deconvolution. Neuroimage 19:200-207. CrossRef Medline

Good BH, de Montjoye YA, Clauset A (2010) Performance of modularity maximization in practical contexts. Phys Rev E Stat Nonlin Soft Matter Phys 81:046106. CrossRef Medline

Gordon EM, Breeden AL, Bean SE, Vaidya CJ (2014) Working memoryrelated changes in functional connectivity persist beyond task disengagement. Hum Brain Mapp 35:1004-1017. CrossRef Medline

Greicius MD, Krasnow B, Reiss AL, Menon V (2003) Functional connectivity in the resting brain: a network analysis of the default mode hypothesis. Proc Natl Acad Sci U S A 100:253-258. CrossRef Medline

Harrison BJ, Yücel M, Fornito A, Wood SJ, Seal ML, Clarke K, Pantelis C (2007a) Characterizing anterior cingulate activation in chronic schizo- phrenia: a group and single-subject fMRI study. Acta Psychiatr Scand 116:271-279. CrossRef Medline

Harrison BJ, Yücel M, Pujol J, Pantelis C (2007b) Task-induced deactivation of midline cortical regions in schizophrenia assessed with fMRI. Schizophr Res 91:82-86. CrossRef Medline

Harrison BJ, Pujol J, Contreras-Rodríguez O, Soriano-Mas C, López-Solà M, Deus J, Ortiz H, Blanco-Hinojo L, Alonso P, Hernández-Ribas R, Cardoner N, Menchón JM (2011) Task-induced deactivation from rest extends beyond the default mode brain network. PLoS One 6:e22964. CrossRef Medline

Houdé O, Rossi S, Sandrine R, Lubin A, Joliot M (2010) Mapping numerical processing, reading, and executive functions in the developing brain: an fMRI meta-analysis of 52 studies including 842 children. Dev Sci 13:876885. CrossRef Medline

Karlsson S, Nyberg L, Karlsson P, Fischer H, Thilers P, Macdonald S, Brehmer Y, Rieckmann A, Halldin C, Farde L, Báckman L (2009) Modulation of striatal dopamine D1 binding by cognitive processing. Neuroimage 48 : 398-404. CrossRef Medline

Kelly AM, Uddin LQ, Biswal BB, Xavier Castellanos FX, Milham MP (2008) Competition between functional brain networks mediates behavioral variability. Neuroimage 39:527-537. CrossRef Medline

Lancichinetti A, Fortunato S (2012) Consensus clustering in complex networks. Sci Rep 2:336. CrossRef Medline

Leech R, Kamourieh S, Beckmann CF, Sharp DJ (2011) Fractionating the default mode network: distinct contributions of the ventral and dorsal posterior cingulate cortex to cognitive control. J Neurosci 31:3217-3224. CrossRef Medline

Luna B (2009) Developmental changes in cognitive control through adolescence. Adv Child Dev Behav 37:233-278. CrossRef Medline

Luna B, Padmanabhan A, O'Hearn K (2010) What has fMRI told us about the development of cognitive control through adolescence? Brain Cogn 72:101-113. CrossRef Medline

Margulies DS, Vincent JL, Kelly C, Lohmann G, Uddin LQ, Biswal BB, Villringer A, Castellanos FX, Milham MP, Petrides M (2009) Precuneus shares intrinsic functional architecture in humans and monkeys. Proc Natl Acad Sci U S A 106:20069-20074. CrossRef Medline

Matthews SC, Simmons AN, Strigo I, Jang K, Stein MB, Paulus MP (2007) Heritability of anterior cingulate response to conflict: an fMRI study in female twins. Neuroimage 38:223-227. CrossRef Medline

Meilă M (2007) Comparing clusterings-an information based distance. J Multivar Anal 98:873-895. CrossRef

Menon V, Uddin LQ (2010) Saliency, switching, attention and control: a network model of insula function. Brain Struct Funct 214:655-667. CrossRef Medline

Miller EK (2000) The prefrontal cortex and cognitive control. Nat Rev Neurosci 1:59-65. CrossRef Medline

Moffitt TE, Arseneault L, Belsky D, Dickson N, Hancox RJ, Harrington H, Houts R, Poulton R, Roberts BW, Ross S, Sears MR, Thomson WM, Caspi A (2011) A gradient of childhood self-control predicts health, wealth, and public safety. Proc Natl Acad Sci U S A 108:2693-2698. CrossRef Medline

Munoz DP, Everling S (2004) Look away: the anti-saccade task and the voluntary control of eye movement. Nat Rev Neurosci 5:218-228. CrossRef Medline

Nee DE, Wager TD, Jonides J (2007) Interference resolution: insights from a meta-analysis of neuroimaging tasks. Cogn Affect Behav Neurosci 7:1-17. CrossRef Medline

Newman ME (2006) Modularity and community structure in networks. Proc Natl Acad Sci U S A 103:8577-8582. CrossRef Medline

Pardo JV, Pardo PJ, Janer KW, Raichle ME (1990) The anterior cingulate cortex mediates processing selection in the Stroop attentional conflict paradigm. Proc Natl Acad Sci U S A 87:256-259. CrossRef Medline

Paus T, Keshavan M, Giedd JN (2008) Why do many psychiatric disorders emerge during adolescence? Nat Rev Neurosci 9:947-957. CrossRef Medline

Persson J, Lustig C, Nelson JK, Reuter-Lorenz PA (2007) Age differences in deactivation: a link to cognitive control? J Cogn Neurosci 19:1021-1032. CrossRef Medline

Power JD, Cohen AL, Nelson SM, Wig GS, Barnes KA, Church JA, Vogel AC, Laumann TO, Miezin FM, Schlaggar BL, Petersen SE (2011) Functional network organization of the human brain. Neuron 72:665-678. CrossRef Medline 
Rieckmann A, Karlsson S, Karlsson P, Brehmer Y, Fischer H, Farde L, Nyberg L, Bäckman L (2011) Dopamine D1 receptor associations within and between dopaminergic pathways in younger and elderly adults: links to cognitive performance. Cereb Cortex 21:2023-2032. CrossRef Medline

Roberts KL, Hall DA (2008) Examining a supramodal network for conflict processing: a systematic review and novel functional magnetic resonance imaging data for related visual and auditory stroop tasks. J Cogn Neurosci 20:1063-1078. CrossRef Medline

Rubinov M, Sporns O (2011) Weight-conserving characterization of complex functional brain networks. Neuroimage 56:2068-2079. CrossRef Medline

Saad ZS, Gotts SJ, Murphy K, Chen G, Jo HJ, Martin A, Cox RW (2012) Trouble at rest: how correlation patterns and group differences become distorted after global signal regression. Brain Connect 2:25-32. CrossRef Medline

Schneider KA, Kastner S (2009) Effects of sustained spatial attention in the human lateral geniculate nucleus and superior colliculus. J Neurosci 29: 1784-1795. CrossRef Medline

Seminowicz DA, Davis KD (2007) Interactions of pain intensity and cognitive load: the brain stays on task. Cereb Cortex 17:1412-1422. CrossRef Medline

Shehzad Z, Kelly AM, Reiss PT, Gee DG, Gotimer K, Uddin LQ, Lee SH, Margulies DS, Roy AK, Biswal BB, Petkova E, Castellanos FX, Milham MP (2009) The resting brain: unconstrained yet reliable. Cereb Cortex 19: 2209-2229. CrossRef Medline

Shulman GL, Fiez JA, Corbetta M, Buckner RL, Miezin FM, Raichle E, Petersen Se (1997) Common blood flow changes across visual tasks: II. Decreases in cerebral cortex. J Cogn Neurosci 9:648-663. CrossRef Medline

Smith SM, Fox PT, Miller KL, Glahn DC, Fox PM, Mackay CE, Filippini N, Watkins KE, Toro R, Laird AR, Beckmann CF (2009) Correspondence of the brain's functional architecture during activation and rest. Proc Natl Acad Sci U S A 106:13040-13045. CrossRef Medline

Spreng RN, Schacter DL (2012) Default network modulation and largescale network interactivity in healthy young and old adults. Cereb Cortex 22:2610-2621. CrossRef Medline

Stern ER, Welsh RC, Fitzgerald KD, Taylor SF (2009) Topographic analysis of individual activation patterns in medial frontal cortex in schizophrenia. Hum Brain Mapp 30:2146-2156. CrossRef Medline

Uddin LQ, Supekar KS, Ryali S, Menon V (2011) Dynamic reconfiguration of structural and functional connectivity across core neurocognitive brain networks with development. J Neurosci 31:18578-18589. CrossRef Medline

van den Heuvel M, Mandl R, Hulshoff Pol H (2008) Normalized cut group clustering of resting-state fMRI data. PLoS One 3:e2001. CrossRef Medline

Vincent JL, Kahn I, Snyder AZ, Raichle ME, Buckner RL (2008) Evidence for a frontoparietal control system revealed by intrinsic functional connectivity. J Neurophysiol 100:3328-3342. CrossRef Medline

Wechsler D (1999) Wechsler Abbreviated Scale of Intelligence (WASI). San Antonio, TX: Harcourt.

Weissman DH, Roberts KC, Visscher KM, Woldorff MG (2006) The neural bases of momentary lapses in attention. Nat Neurosci 9:971-978. CrossRef Medline

Whittle S, Yap MB, Yücel M, Fornito A, Simmons JG, Barrett A, Sheeber L, Allen NB (2008) Prefrontal and amygdala volumes are related to adolescents' affective behaviors during parent-adolescent interactions. Proc Natl Acad Sci U S A 105:3652-3657. CrossRef Medline

Yücel M, Harrison BJ, Wood SJ, Fornito A, Clarke K, Wellard RM, Cotton S, Pantelis C (2007a) State, trait and biochemical influences on human anterior cingulate function. Neuroimage 34:1766-1773. CrossRef Medline

Yücel M, Harrison B, Wood SJ, Fornito A, Wellard RM, Pujol J, Clarke K, Phillips ML, Kyrios M, Velakoulis D, Pantelis C (2007b) Functional and biochemical alterations of the medial frontal cortex in obsessivecompulsive disorder. Arch Gen Psychiatry 64:946-955. CrossRef Medline

Yücel M, Lubman DI, Harrison BJ, Fornito A, Allen NB, Wellard RM, Roffel K, Clarke K, Wood SJ, Forman SD, Pantelis C (2007c) A combined spectroscopic and functional MRI investigation of the dorsal anterior cingulate region in opiate addiction. Mol Psychiatry 12:611, 691-702. CrossRef Medline

Zalesky A, Fornito A, Bullmore ET (2010) Network-based statistic: identifying differences in brain networks. Neuroimage 53:1197-1207. CrossRef Medline 